\title{
Assessment of microbial populations dynamics in a blue cheese by culturing and denaturing gradient gel electrophoresis (DGGE)
}

\author{
Ángel Alegría ${ }^{1}$, Renata González ${ }^{2}$, Mario Díaz $^{2}$, and Baltasar Mayo ${ }^{1 *}$ \\ ${ }^{1}$ Departamento de Microbiología y Bioquímica, Instituto de Productos Lácteos de Asturias \\ (CSIC), Carretera de Infiesto, s/n, 33300-Villaviciosa, Spain, and ${ }^{2}$ Departamento de \\ Ingeniería Química y Tecnología del Medio Ambiente, Facultad de Químicas, Universidad \\ de Oviedo, C/ Julián Clavería, s/n, 33006-Oviedo, Spain
}

RUNNING TITLE: Culturing and DGGE cheese analysis

Key words:

cheese, blue cheese, microbial populations, cheese microbiology, culture-independent methods, DGGE

*Corresponding author:

Baltasar Mayo, ${ }^{1}$ Departamento de Microbiología y Bioquímica, Instituto de Productos

Lácteos de Asturias (CSIC), Carretera de Infiesto s/n, 33300-Villaviciosa, Spain

Tel.: 34+985892131

Fax: 34+985 892233

E-mail address: baltasar.mayo@ipla.csic.es 


\section{Summary}

2

3 The composition and development of microbial population during the manufacture and

4 ripening of two batches of a blue-veined cheese was examined by culturing and polymerase

5 chain reaction (PCR) denaturing gradient gel electrophoresis (DGGE) (PCR-DGGE). Nine

6 selective and/or differential media were used to track the cultivable populations of total and

7 indicator microbial groups. For PCR-DGGE, the V3 hyper variable region of the bacterial

8 16S rRNA gene and the eukaryotic D1 domain of 28S rDNA were amplified with universal

9 primers, specific for prokaryotes and eukaryotes, respectively. Similarities and differences

10 between the results obtained by the culturing and the molecular method were recorded for

11 some populations. Culturing analysis allows minority microbial groups (coliforms,

12 staphylococci) to be monitored, although in this work PCR-DGGE identified a population of

13 Streptococcus thermophilus that went undetected by culturing. These results show that the

14 characterization of the microbial populations interacting and evolving during the

15 cheesemaking process is improved by combining culturing and molecular methods. 


\section{Introduction}

The microbial characterization of dairy products has traditionally been performed using culture dependent techniques employing non-selective, selective and differential media; this allows the composition and development of majority and indicator populations present during manufacturing and ripening to be monitored. However, a vast array of culture-independent molecular methods is now being used for the microbial typing of food and food fermentations $[10,11]$. Molecular methods overcome culture-associated drawbacks such as the low specificity of selective media and the inability to enumerate cells in a viable but noncultivable state. Most molecular methods rely on the amplification of rDNA or rRNA sequences by the polymerase chain reaction $(\mathrm{PCR})$ and the subsequent electrophoretic analysis of the amplicons produced [1]. In addition, they are less laborious, faster and cheaper than culture-based analyses. However, these techniques are not free from bias nor without limitations [20] and are generally thought of as complementary to conventional methods, providing a more precise microbial picture of food ecosystems.

Denaturing gradient gel electrophoresis (DGGE) and its relative, temperature gradient gel electrophoresis (TGGE), were developed to analyse microbial communities based on sequence-specific distinctions of 16S rRNA amplicons produced by PCR [15]. Separation is based on the reduced electrophoretic mobility of partially melted double-stranded DNA molecules in polyacrylamide gels with a linear gradient of denaturing agents (urea and formamide) or temperature. A GC clamp of around $50 \mathrm{bp}$ is attached to the 5'end of one of the primers, preventing the two DNA strands from undergoing complete disassociation. If the total DNA of a microbial community is used in PCR amplification these techniques can provide the profile of the genetic diversity of the dominant populations. If total RNA is used 
40 instead, the profiles reveal the metabolically-active populations [14]. Both PCR-DGGE and

41 PCR-TGGE have recently been used to study different microbial aspects of food-related

42

61

62 environments [7], including the diversity and dynamics of microorganisms present during cheese manufacture and ripening $[4,5,6,8,16]$.

The present paper reports the use of plate count methods and PCR-DGGE analysis for studying microbial development, diversity and evolution during the manufacture and ripening of a blue cheese made from pasteurized milk inoculated with commercial starters (mesophilic lactic acid bacteria as acidifiers and Penicillium roqueforti spores as the ripening culture). The aim of this work was to compare the results obtained with the culture-dependent and cultureindependent methods, assessing the advantages and drawbacks of each.

\section{Materials and Methods}

Cheese manufacture and sampling conditions

Two batches of blue-veined cheese were manufactured from pasteurized milk under standard conditions [9]. Acidifying starters (Flora Danica) and ripening cultures (Penicillium roqueforti strains PR3 and PRG3) were purchased from Chr. Hansen (Hørsholm, Denmark). Milk, curd and cheese were sampled according to FIL-IDF standard 50B and transported to the laboratory under refrigerated conditions. Culturing analyses were performed on the day of sampling. For the isolation of DNA, milk, curd and cheese samples were stored at $-20{ }^{\circ} \mathrm{C}$ until required.

Determination of microbial composition by plate counting 
Ten milliliters of milk or $10 \mathrm{~g}$ of curd and cheese were homogenized with $90 \mathrm{~mL}$ of a 2 $\%(w / v)$ sodium citrate solution at $45^{\circ} \mathrm{C}$ in a Colworth Stomacher 400 (Seward Ltd., London, UK) (for 3 x 1 min). Ten-fold serial dilutions were made in Maximum Recovery Diluent (Scharlab, Barcelona, Spain) and plated in duplicate onto general and selective media, as

67 follows.

68 Aerobic mesophilic counts. Aerobic mesophilic bacteria were counted on Plate Count Agar supplemented with $0.1 \%$ skimmed milk (PCA; Merck, Darmstadt, Germany) after $72 \mathrm{~h}$ of incubation in aerobiosis at $30^{\circ} \mathrm{C}$.

Lactococci: Lactococci were grown on M17 agar (Scharlab) and enumerated after $48 \mathrm{~h}$ of 72 incubation at $30^{\circ} \mathrm{C}$.

73 Lactobacilli. Lactobacilli were grown on de Man, Rogosa and Sharpe agar (MRS; Merck), 74 adjusted to $\mathrm{pH}=5.4$ and enumerated after $72 \mathrm{~h}$ of incubation at $32{ }^{\circ} \mathrm{C}$ in a $5 \% \mathrm{CO}_{2}$ enriched 75 incubator.

Leuconostoc spp. Dextran-producing leuconostocs were grown on Mayeux, Sandine and

77 Elliker agar (MSE; Biokar Diagnostics, Beauvais, France) and enumerated after five days of 78 incubation at $25^{\circ} \mathrm{C}$.

79 Enterococci. Enterococci were grown on Slanetz and Bartley agar (S-B; Merck) and enumerated after $24 \mathrm{~h}$ of incubation at $44^{\circ} \mathrm{C}$.

81 Enterobacteria and coliforms. Enterobacteria and coliforms were grown on Violet Red Bile

82 Glucose agar (VRBG) and Violet Red Bile Lactose agar (VRBL) (both from Merck)

83 respectively, using the pour-plate and overlay technique. In brief, dilutions were mixed with

$8415 \mathrm{~mL}$ of agar and poured onto Petri dishes. After solidification, a second agar layer of $10 \mathrm{~mL}$ was added. Bacteria were enumerated after $48 \mathrm{~h}$ of incubation at $30^{\circ} \mathrm{C}$. 
86 Staphylococci. Dilutions were grown on Baird-Parker agar (B-P; Merck) supplemented with

87 egg yolk tellurite solution (Merck). Black colonies with or without egg yolk clearing were

88

89

90

91

92

93

94

95

96

97

101

102

103

104

105

106

107

108

109 recorded after $24 \mathrm{~h}$ of incubation at $37^{\circ} \mathrm{C}$.

Yeasts and moulds. Dilutions of milk, curd and cheese samples were plated on Yeast-Extract

Glucose Chloramphenicol agar (YGC; Merck). Yeasts and moulds were independently recorded after 3-5 days of incubation at $25^{\circ} \mathrm{C}$.

Except for the YGC plates, $100 \mu \mathrm{g} / \mathrm{mL}$ of cycloheximide (Merck) was added to all enumeration media to inhibit the growth of moulds and yeasts.

\section{PCR-DGGE analysis}

Extraction of total microbial DNA.

Milk, cheese and starter samples homogenized in $2 \%$ sodium citrate were used for the isolation of total microbial DNA. DNA extraction was accomplished using a commercial kit (QIAamp DNA Stool Mini Kit; Quiagen, GmbH, Hilden, Germany), following the supplier's recommendations.

PCR amplification.

Total DNA was used as a template in PCR amplification of the V3 region of the bacterial 16S rRNA gene using the universal primers F357 (5'-TACGGGAGGCAGCAG-3'), to which a 39 bp GC sequence was linked to give rise to GC-F357), and R518 (5'-

ATTACCGCGGCTGCTGG-3') [14]. The D1 domain of the 28S rRNA fungal gene was amplified using primers GC-NL1 (5'-GCCATATCAATAAGCGGAGGAAAAG-3') and LS2 (5'-ATTCCCAAACAACTCGACTC-3') [3]. PCR was performed in a $50 \mu$ l volume using a Taq-DNA polymerase master mix $\left(75 \mathrm{mM}\right.$ Tris- $\mathrm{HCl} \mathrm{pH}=8.5,20 \mathrm{mM}\left(\mathrm{NH}_{4}\right)_{2} \mathrm{SO}_{4}, 1.5 \mathrm{mM}$ 
$110 \mathrm{MgCl}_{2}, 0.1 \%$ Tween $20^{\circledR}, 0.2 \mathrm{mM}$ of each dNTP, and 1.25 units Taq polymerase (Ampliqon 111 ApS, Skovlunde, Denmark), with $100 \mathrm{ng}$ of extracted DNA and $0.2 \mathrm{mM}$ of each primer. The 112 amplification conditions for prokaryotic and eukaryotic sequences were those described by 113 Muyzer et al. [14] and Cocolin et al. [3], respectively.

Electrophoresis conditions.

DGGE was performed using a DCode apparatus (Bio-Rad, Richmond, CA., USA) at $60{ }^{\circ} \mathrm{C}$,

117 employing $8 \%$ polyacrylamide gels with a denaturing range of 40-60\% for bacteria and 30$50 \%$ for fungi. Electrophoresis was performed at $75 \mathrm{~V}$ for $17 \mathrm{~h}$ and at $130 \mathrm{~V}$ for $4.5 \mathrm{~h}$ for bacterial and fungal amplifications respectively. Bands were visualized by staining with 0.5 $\mu \mathrm{g} / \mathrm{mL}$ ethidium bromide (Sigma-Aldrich, St. Louis, MO, USA).

Identification of PCR-DGGE bands.

DNA bands in the polyacrylamide gels were assigned to species by either comparison with a control ladder of known strains [8], or by sequencing and comparison of the sequences after the same species (Stackebrandt and Goebel, 1994).

\section{Results and Discussion}

Microbial counts using conventional plate count techniques

Tables 1 and 2 show the enumeration results for total and indicator populations in the two batches over manufacturing and ripening. As expected for a cheese made from 
134 pasteurized milk, Enterobacteriaceae, coliforms, enterococci and staphylococci counts were

135 always in low numbers, though small variations between batches were recorded. No

136 Staphylococcus aureus strains were detected. Lactococci were found in the largest numbers;

137 in M17 the numbers recorded usually matched those of total bacterial aerobic counts in PCA,

138 reaching over $10^{9}$ colony forming units (CFU)/g between day 3 and day 7 (Tables 1 and 2).

139 Lactobacilli grew from low numbers in milk up to $10^{8} \mathrm{CFU} / \mathrm{g}$ by day 7 , although their final

140 numbers were one log unit lower than those of lactococci. Both lactococci and lactobacilli

141 populations showed a slight decline from day 15 onwards. The yeast and mould populations

142 increased throughout ripening, approaching or surpassing (depending on the batch) $10^{7} \mathrm{CFU} / \mathrm{g}$

143 of cheese. Because of their different morphology on the YGC plates, yeasts and moulds could

144 be recorded separately (Table 1). Two P. roqueforti strains were distinguishable from one

145 another owing to their different colored mycelia (PR3 dark green, PRG3 pale gray). Yeasts

146 started at similar numbers in both batches, however, counts for batch 1 increased over those

147 for batch 2 (maximum $6.1 \times 10^{7}$ and $5.75 \times 10^{5} \mathrm{CFU} / \mathrm{g}$ in batches 1 and batch 2 respectively).

148 Variability in the cultivable microbial populations of dairy products is well known, even

149 when they are made from pasteurized milk [2, 19]. Different initial microbial loads (in

150 numbers and types) and post-pasteurization contamination may be responsible for the

151 majority of the differences observed in most studies.

153 Microbial composition and dynamics as revealed by PCR-DGGE

$154 \quad$ Figures 1 and 2 show the composition and dynamics of the prokaryotic and eukaryotic

155 populations in batch 1 and batch 2 determined by PCR-DGGE. Panel A in both figures

156 corresponds to the DGGE patterns of batch 1, Panel B to those of batch 2. The profiles of the

157 cheese batches were relatively simple compared to the complex DGGE patterns of cheeses 
made of raw milk $[4,8,16]$. More bands were seen in the two milk samples, though most of them proved to be very faint. The intensity of an individual band is assumed to be a semi-

quantitative measure of the abundance of the corresponding microorganism in the original population [14]. In addition to bands for L. lactis (band 4) and Lactobacillus plantarum (band 1), bands corresponding to Weisella cibaria (band 2) and Actinobacterium spp. (band 3) were found in the two milk samples (Line 1 in Figures 1A and 1B). As for the cheeses, three and four different bacterial bands were observed in batch 1 and 2 respectively. L. lactis was the most prominent band (band 4) in both. In the cheese samples, bands of lactococci were always accompanied by two bands identified as Streptococcus thermophilus (bands 5 and 6). In addition, in batch 2 another band located between bands 4 and 5 (band 7) was observed throughout manufacture and cheese ripening . This band was also present in the PCR-DGGE profile of the Flora Danica starter (Line S in Figure 1B). The sequence of this band was identical to that of L. lactis (band g). The DGGE pattern of Flora Danica produced two additional bands in the uppermost part of the gel (bands 8 and 9), the DNA sequence of which showed around $98 \%$ similarity to L. lactis sequences. The presence of double bands corresponding to a single species may be due to heterogeneous copies of rRNA operons, a well-established limiting factor of the PCR-DGGE technique, but also to other artifacts related to the melting and re-association properties of related sequences $[12,17]$.

Figure 2 shows the DGGE profiles obtained with the primers for amplifying eukaryotic sequences. Five different bands were observed among the samples of the two batches. All were identified by isolation, reamplification, sequencing and comparison against sequences in databases. The sequences of two bands (band 1 and 2) were identical to those known for Debaryomyces hansenii; these were present in all samples of batch 1 after day 7 (except on day 15; line 5 in Figure 2A), while only a faint band was observed in the 15 day sample in 
182 batch 2. In the lower part of the gels, three patent bands appeared in samples of both batches

183 from day 7 onwards (bands 3, 4, and 5). The sequence of these bands matched those of $P$.

184 roqueforti. As expected, PCR-DGGE analysis of Flora Danica with the eukaryotic primers

185 gave no bands (Line $\mathrm{S}$ in Figure 2B).

186 The bands of both the prokaryotic and eukaryotic populations remained the same

187 (qualitatively and quantitatively) during the entire cheese-making process. Of note is the

188 absence in cheese of bands corresponding to bacteria from milk (except for that of L. lactis, a

189 bacterium also present in the starter). Microorganisms in the pasteurized milk may be in a

190 viable but non-cultivable state, and then do not progress into the cheese, or if they grow only

191 small numbers are reached as compared to those obtained after the addition of starters.

192 Although non declared, the $S$. thermophilus population detected may have come from the

193 Flora Danica; as at least one of the two bands observed in the cheese samples was also

194 detected for this undefined starter culture (the lowest band in line $\mathrm{S}$ in Figure 1B).

195

196

197

198

199

200

201

202

203

204

205

Comparison of plate count and PCR-DGGE results

The results obtained by culturing and DGGE showed similarities as well as patent differences. The heterogeneous distribution of microorganisms within the samples is a widely known cause of variation between and among culturing and DGGE results. The molecular method proved to be faster and cheaper in terms of running costs. Further, the analysis of the diversity and development of the microbial populations in the two batches over manufacturing and ripening (14 samples) was performed in a couple of weeks instead of the four months needed to finish conventional culturing analysis.

Intriguing is the differential results obtained for the lactobacilli with the two techniques.

By conventional culturing, these were shown to form part of the majority populations, 
reaching numbers similar to those of lacococci (as in batch 2, day 60). However, although bands related to lactobacilli were detected in milk, they were never detected in cheese.

\section{Conclusion}

Classic culturing and molecular methods have been repeatedly reported to provide complementary results; this affirmation is strengthened by the results of the present work. 
230 Both plate counting and PCR-DGGE analysis can be used for identifying and tracking

231 majority populations throughout cheese manufacture and ripening, and both have their own

232 advantages and drawbacks. They are both easy to perform, but the PCR-DGGE technique still

233 requires dedicated equipment and reagents (thermocycler, nucleotides, polymerase, DGGE

234 apparatus, etc.). Thus, the choice of using one or another ultimately depends on the purpose of

235 the study if all necessary materials and instruments are available. However, the combination

236 of the two undoubtedly improves the microbial characterization of the cheese-making process.

237

238

239

240

241

242

243

244

245

246

247

248

249

250

251

252

253

\section{Acknowledgements}

This research was supported by projects to B.M. from MICINN (AGL2007-61869-ALI), and to M.D. from FICYT (PC07-05). Á.A. awarded a scholarship of the Severo Ochoa program from FICYT (BP08-053).

\section{References}

1. Amann RI, Ludwingg W, Schleifer KF (1995) Phylogenetic identification and in situ detection of individual microbial cells without cultivation. Microbiol Rev 59:143-169

2. Beresford TP, Fitzsimons NA, Brennan NL, Cogan TM (2001) Recent advances in cheese microbiology. Int Dairy J 11:259-274

3. Cocolin L, Aggio D, Manzano M, Cantoni C, Comi G (2002) An application of PCRDGGE analysis to profile the yeast populations in raw milk. Int Dairy J 12:407-411

4. Coppola S, Blaiotta G, Ercolini D, Moschetti G (2001) Molecular evaluation of microbial diversity in different types of Mozzarella cheese. J Appl Microbiol 90:414-420

5. Ercolini D, Hill PJ, Dood CER (2003) Bacterial community structure and location in Stilton cheese. Appl Environ Microbiol 69:3540-3548 
254 6. Ercolini D, Mauriello G, Blaiotta G, Moschetti G, Coppola S (2004) PCR-DGGE

255 fingerprints of microbial succession during a manufacture of traditional water buffalo mozzarella cheese. J Appl Microbiol 96:263-270

7. Ercolini D (2004) PCR-DGGE fingerprinting: novel strategies for detection of microbes in food. J Microbiol Methods 56:297-314

8. Flórez AB, Mayo B (2006) Microbial diversity and succession during the manufacture and ripening of traditional, Spanish, blue-veined Cabrales cheese, as determined by PCRDGGE. Int J Food Microbiol 110:165-171

9. Fox PF, Guinee, TP, Cogan, TM, McSweeney, TP (2000) Fundamentals of Cheese Science. Gaithersburg, Maryland, Aspen Publishers Inc, pp 415-418

10. Giraffa G, Neviani E (2001) DNA-based, culture-independent strategies for evaluating microbial communities in food-associated ecosystems. Int J Food Microbiol 67:19-34

11. Jany JL, Barbier g (2008) Culture-independent methods for identifying microbial communities in cheese. Food Microbiol 25:839-848

12. Kisand V, Wikner J (2003) Limited resolution of 16S rDNA DGGE caused by melting properties and closely related DNA sequences. J Microbiol Methods 54:1183-191

13. Lodics TA, Steenson LR (1989) Characterization of bacteriophages and bacteria indigenous to a mixed-strain cheese starter. J Dairy Sci 73:2685-2696

14. Muyzer G, de Waal EC, Uitterlinden AG (1993) Profiling of complex microbial populations by denaturing gradient gel electrophoresis analysis of polymerase chain reaction-amplified genes encoding for 16S rRNA. Appl Environ Microbiol 59:695-700

15. Muyzer, G, Smalla K (1998) Application of denaturing gradient gel electrophoresis (DGGE) and temperature gradient gel electrophoresis (TGGE) in microbial ecology. Antonie van Leeuwenhoek 73:127-141 
278 16. Randazzo CL, Torriani S, Akkermans ALD, de Vos WM, Vaughan EE (2002) Diversity, 279 dynamics, and activity of bacterial communities during production of an artisanal Sicilian 280 cheese as evaluated by 16S rRNA analysis. Appl Environ Microbiol 68:1882-1892 281 17. Stackebrandt E, Goebel BM (1994) Taxonomic note: a place for DNA-DNA reassociation 282 and 16S rRNA sequence analysis in the present species definition in bacteriology. Int J 283 Syst Bacteriol 44:846-849

284 18. von Wintzingerode F, Göbel UB, Stackebrandt E (1997) Determination of microbial 285 diversity in environmental samples: pitfalls of PCR-based rRNA analysis. FEMS $286 \quad$ Microbiol Rev 21:213-229

287 19. Walter J, Tannock GW, Tilsala-Timisjarvi A, Rodtong S, Loach DM, Munro K, 288 Alatossava A (2001) Detection of gastrointestinal Lactobacillus species by using 289 denaturing gradient gel electrophoresis and species-specific primers. Appl Environ $290 \quad$ Microbiol 66:297-303

291 20. Wouters JTM, Ayad EHE, Hugenholtz J, Smit G (2002) Microbes from raw milk for 292 fermented dairy products. Int Dairy J 12:91-109 
293 Table 1.- Bacterial counts in log10 CFU mL or g determined in milk and cheese samples of batch

2941 during cheesemaking and ripening.

295

\begin{tabular}{|c|c|c|c|c|c|c|c|}
\hline \multirow{2}{*}{$\begin{array}{l}\text { Microbial group } \\
\text { (counting medium) }\end{array}$} & \multicolumn{7}{|c|}{ Stage of manufacture or ripening } \\
\hline & Milk & Curd & 3 day & 7 day & $\begin{array}{c}15 \\
\text { day }\end{array}$ & $\begin{array}{c}30 \\
\text { day }\end{array}$ & $\begin{array}{c}60 \\
\text { day }\end{array}$ \\
\hline Total aerobic counts (PCA) & 5.01 & 8.53 & 9.13 & 9.28 & 8.54 & 8.41 & 8.58 \\
\hline Lactococci (M17) & 5.80 & 8.41 & 9.11 & 9.20 & 8.58 & 8.37 & 8.57 \\
\hline Lactobacilli (MRS, pH 5.4) & 3.03 & 7.26 & 8.37 & 8.49 & 8.18 & 7.74 & 7.48 \\
\hline Leuconostoc (MSE) & $<1$ & $<4.00$ & $<4.00$ & $<4.00$ & $<4.00$ & $<4.00$ & $<4.00$ \\
\hline Enterobacteriaceae (VRBG) & $<1$ & 3.88 & 3.89 & 2.40 & $<2.00$ & 2.00 & 2.00 \\
\hline Coliforms (VRBL) & $<1$ & 4.13 & 3.90 & 2.30 & $<2.00$ & 2.00 & 2.00 \\
\hline Enterococci (S-B) & $<1$ & $<2.00$ & $<2.00$ & $<2.00$ & $<2.00$ & $<2.00$ & 5.67 \\
\hline Staphylococci (B-P) & 1.48 & 2.18 & 2.74 & 2.00 & $<2.00$ & $<2.00$ & 2.30 \\
\hline Yeasts (YGC) & $<1$ & 2.00 & $<3.00$ & 8.66 & 5.94 & 7.79 & 7.72 \\
\hline P. roqueforti PR3 (YGC) & $<1$ & 3.74 & 3.83 & $<4.00$ & $<3.00$ & $<4.00$ & 4.30 \\
\hline P. roqueforti PRG3 (YGC) & $<1$ & 4.08 & 4.26 & 7.64 & 6.88 & 6.64 & 5.81 \\
\hline
\end{tabular}

297 The symbol < is used to indicate that numbers were lower than the detection limit. 
298 Table 2.- Bacterial counts in log10 CFU $\mathrm{mL}$ or $\mathrm{g}$ determined in milk and cheese samples of batch 2 299 during cheesemaking and ripening.

300

\begin{tabular}{|c|c|c|c|c|c|c|c|}
\hline \multirow{2}{*}{$\begin{array}{l}\text { Microbial group } \\
\text { (counting medium) }\end{array}$} & \multicolumn{7}{|c|}{ Stage of manufacture or ripening } \\
\hline & Milk & Curd & 3 day & 7 day & $\begin{array}{c}15 \\
\text { day }\end{array}$ & $\begin{array}{c}30 \\
\text { day }\end{array}$ & $\begin{array}{c}60 \\
\text { day }\end{array}$ \\
\hline Total aerobic counts (PCA) & 4.60 & 7.46 & 9.13 & 9.25 & 9.09 & 8.95 & 8.60 \\
\hline Lactococci (M17) & 3.91 & 7.75 & 9.35 & 9.35 & 9.36 & 9.02 & 8.48 \\
\hline Lactobacilli (MRS, pH 5.4) & $<1$ & 6.90 & 8.39 & 8.39 & 7.71 & 8.43 & 8.40 \\
\hline Leuconostoc (MSE) & $<1$ & $<4.00$ & $<4.00$ & $<4.00$ & $<4.00$ & $<4.00$ & $<4.00$ \\
\hline Enterobacteriaceae (VRBG) & $<1$ & 2.30 & 2.60 & $<2.00$ & $<2.00$ & $<2.00$ & $<2.00$ \\
\hline Coliforms (VRBL) & $<1$ & 2.30 & 2.78 & $<2.00$ & $<2.00$ & $<2.00$ & $<2.00$ \\
\hline Enterococci (S-B) & $<1$ & $<2.00$ & $<2.00$ & $<2.00$ & $<2.00$ & $<2.00$ & $<2.00$ \\
\hline Staphylococci (B-P) & $<1$ & 2.48 & $<2.00$ & 2.00 & $<2.00$ & 2.00 & $<2.00$ \\
\hline Yeasts (YGC) & $<1$ & 2.00 & $<3.00$ & $<3.00$ & 5.76 & 5.00 & $<5.00$ \\
\hline P. roqueforti PR3 (YGC) & $<1$ & 3.65 & 3.70 & 3.60 & 4.95 & $<5.00$ & $<5.00$ \\
\hline P. roqueforti PRG3 (YGC) & $<1$ & 3.93 & 4.18 & 4.11 & 6.74 & 6.96 & 6.53 \\
\hline
\end{tabular}

301

302 The symbol $<$ is used to indicate that numbers were lower than the detection limit. 
304 Figure 1.- DGGE profiles of microbial populations from cheese during manufacture and

305 ripening, using amplicons of the V3 variable region of the bacterial 16S rRNA gene. Lanes

306 Ma and Mb contain combined amplicons of identified strains used as controls; a) Lactococcus

307 garvieae, b) Lactobacillus plantarum, c) Leuconostoc mesenteroides, d) Streptococcus

308 parauberis. e) Enterococcus faecium, f) Enterococcus faecalis, g) Lactococcus lactis, h)

309 Escherichia coli, i) Lactobacillus paracasei. Panel A contains samples from the first batch.

310 Sample 1: milk; 2: curd; 3, 4, 5, 6 and 7: cheese at 3, 7, 15, 30 and 60 days of ripening. Panel

311 B contains samples from the second batch (same order). Lane S shows the DGGE analysis of

312 a sample of the Flora Danica starter. Key to identified bands: 1) L. plantarum, 2) Weissella

313 cibaria, 3) Actinobacterium spp., 4) L. lactis, 5) and 6) S. thermophilus, 7) L. lactis, 8 and 9)

314 L. lactis-related sequences.

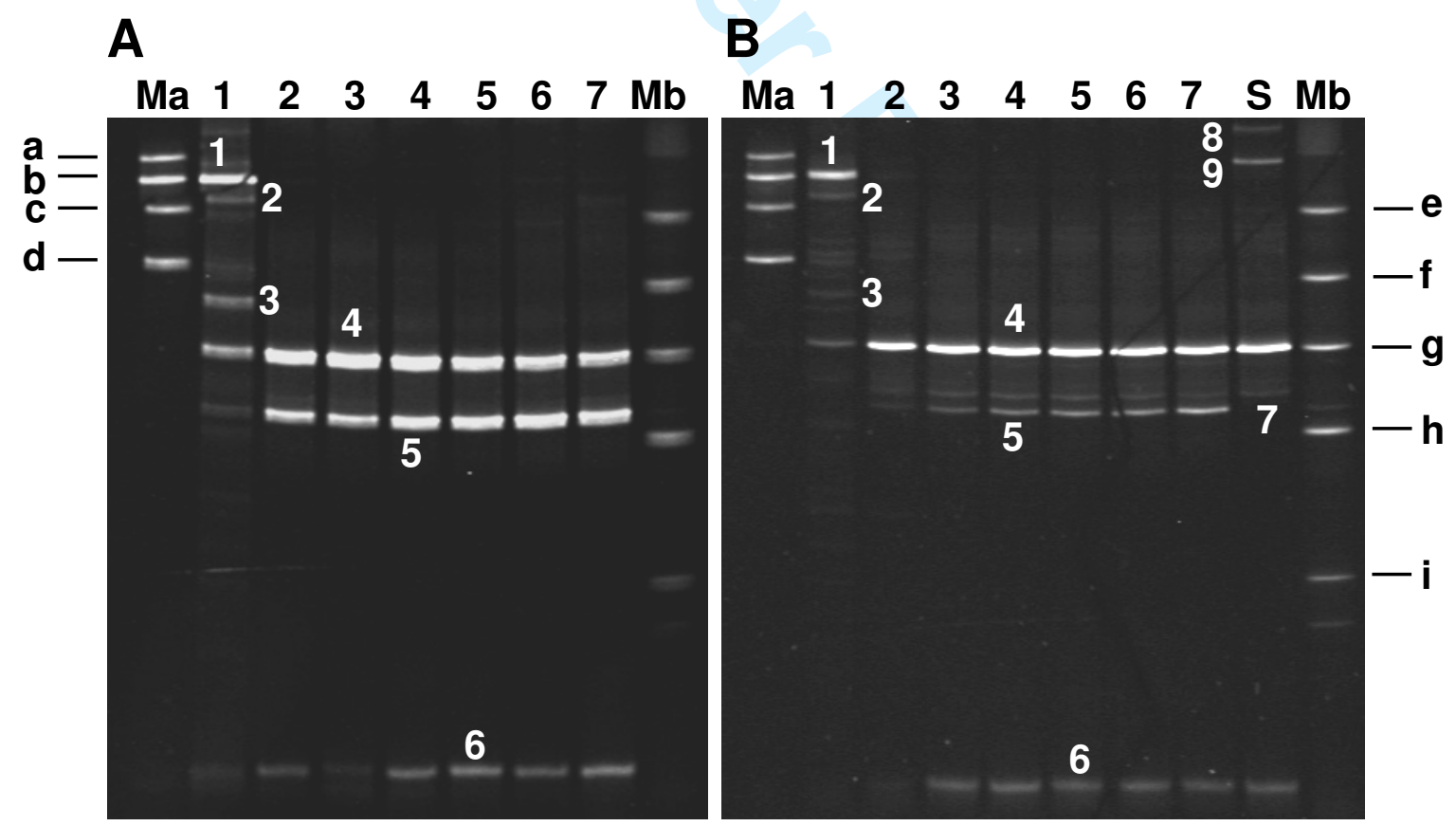


315 Figure 2.- DGGE profiles of moulds and yeasts using a PCR amplicon of the eukaryotic

316 domain D1 of 26S rDNA. Panel A contains samples from the first batch. Sample 1: milk; 2:

317 curd; 3, 4, 5, 6 and 7: cheese at 3, 7, 15, 30 and 60 days of ripening. Panel B contains samples

318 from the second batch. Sample 1: milk; 2: curd; 3, 4, 5, 6 and 7: cheese at 3, 7, 15, 30 and 60

319 days of ripening. Lane S corresponds to the Flora Danica starter. Key to identified bands: 1)

320 and 2) Debaryomyces hansenii, 3, 4, and 5) Penicillium roqueforti.

321

A B

322

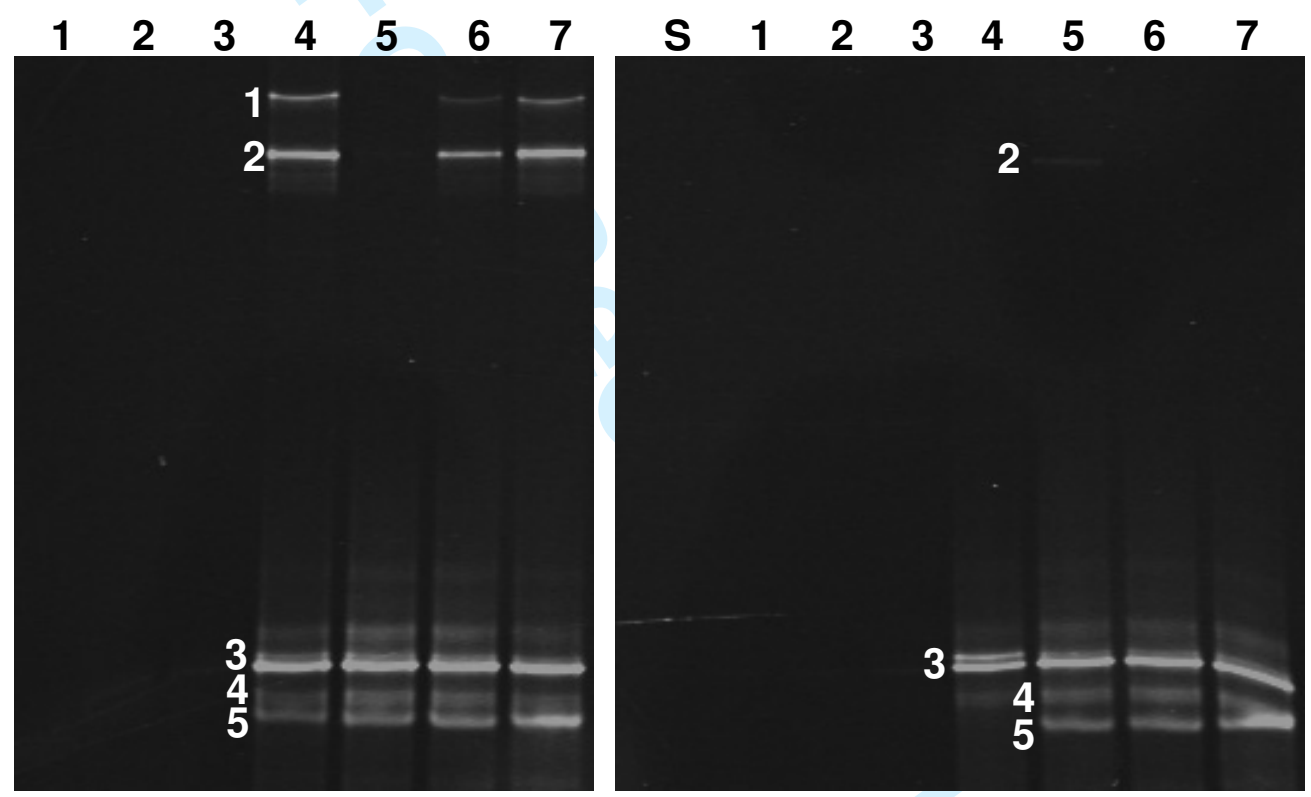

